\title{
Prevalence of Plasmodium falciparum Parasitaemia in Children from Different Breastfeeding Regimens Evaluated by the Deuterium Technique in Southern Benin
}

Yolande Sissinto-Savi de Tove ${ }^{1,2^{\star}}$, Aurore Ogouyemi-Hounto ${ }^{1,2}$, Gilles Cotrell ${ }^{2}$, Jules Maroufou Alao ${ }^{3}$, Amoussa Waliou Hounkpatin ${ }^{4}$, Tornyigah Bernard ${ }^{2}$, Georgia Damien ${ }^{2}$, Atikat Mama ${ }^{2}$, Daniel Kintin ${ }^{1}$, Paul Bankole ${ }^{1}$, Adicatou Adeothy ${ }^{1}$, Clemence Fassinou ${ }^{5}$, David Adomahou ${ }^{2}$, Lionel Adisso ${ }^{2}$, Khalid El Kari ${ }^{7}$, Clement Ahoussinou ${ }^{2}$, Kouassi Marcellin Amoussou Guenou ${ }^{5}$, Nama Gabriel Medoua ${ }^{6}$, Adrian Luty ${ }^{2}$, Tuikue Ndam Nicaise ${ }^{2}$, Achille Massougbodji ${ }^{1,2}$ and Dorothee Kinde Gazard ${ }^{1,2}$

${ }^{1}$ Unit of Teaching and Research in Parasitology-Mycology, University of Abomey Calavi, Benin

${ }^{2}$ The Parasitology Laboratory of the Center for Malaria Research Associated with Pregnancy and Childhood (CERPAGE) and Center for Integrated Control against Malaria (CLIP), Institute for Research and Development (IRD), France

${ }^{3}$ Pediatrics and Medical Genetics Unit, Pediatric Department of the National Hospital, University Center of Cotonou 01BP386 Cotonou, Benin

${ }^{4}$ University of Abomey Calavi, Benin

${ }^{5}$ United Nuclear Medicine Unit, University of Abomey Calavi, Benin

${ }^{6}$ Center for Food and Nutrition Research, IMPM, Yaounde, Cameroon

${ }^{7}$ United Joint Research, Unit in Nutrition and Food-URAC and Unit of Biology and Medical Research, Rabat, Morocco

"Corresponding author: de Tove YSS, Faculty of Health Sciences, University of Abomey Calavi, 01 BP188 Cotonou, Benin, Tel: +22-99426926; E-mail: yolande.sissinto@yahoo.com

Received date: February 23, 2018; Accepted date: March 19, 2018; Published date: March 28, 2018

Copyright: (c) 2018 de Tove YSS, et al. This is an open-access article distributed under the terms of the Creative Commons Attribution License, which permits unrestricted use, distribution and reproduction in any medium, provided the original author and source are credited.

\begin{abstract}
Studies on the health benefits of exclusive breastfeeding (EBF) and susceptibility to malaria have shown contradictory results. These studies often failed to account for dose response effects. The deuterium dilution technique was used to evaluate the breast milk intake, the amount of water from sources other than human milk and the exclusivity of breastfeeding. We determine Plasmodium falciparum parasitaemia prevalence in children aged less than six month according to their breastfeeding regimen and maternal milk dose ingested in southern Benin. We conducted a cross sectional study in the Ouidah Kpomasse Tori-Bossito (OKT) health zone in southern Benin from February to December 2014, an intense transmission season. 115 mothers paired with their children aged from 0 to 6 months were enrolled. The daily human milk was measured through saliva of each mother and child pair (MCP) over a period of 14 days by the technique of deuterium oxide "dose to the mother" using the Fourier Transformed Infrared Spectrometer (FTIR). Malaria parasitaemia in children was determined by quantitative Polymerase Chain Reaction (qPCR) in real time at day 14 and day 28. The average age was 2.3 months. The distribution of Plasmodium falciparum parasitaemia in children was $0.00 \%, 5.08 \%$ and $3.85 \%$ respectively in exclusive, predominant and partial breastfeeding regimens. The mean intake of breast milk was $641.71 \mathrm{~mL}$ per day; $256.75 \mathrm{~mL}$ of water per day was taken from sources other than breastmilk. Adjusted linear regression analysis revealed a significant association between quantity of breastmilk and parasitaemia prevalence; infected children (qPCR positive) had $164.11 \mathrm{~mL}$ of ingested breastmilk per day less than children without parasitaemia $(p=0.00)$. Our findings highlighted the association between the low risk of Plasmodium falciparum parasitaemia and the ingested breastmilk dose. Study with larger numbers of patients would be necessary to confirm this relationship.
\end{abstract}

Keywords Breastfeeding; Deuterium-oxide technique; Plasmodium falciparum parasitaemia, Children

\section{Abbreviations}

KAP: Knowledge, Attitude and Practice;

VL: Visceral Leishmaniasis

\section{Introduction}

Many studies on the health benefits of breastfeeding in developing countries have shown conflicting results. Studies from Uganda and Nigeria suggested that Exclusive Breastfeeding (EBF) had no significant effect on malaria infection risk $[1,2]$. Some studies from Malawi and Congo, found that maternel milk or EBF protected infants from developing malaria [3,4]. However, in all studies, the classification of EBF were based on questionnaires and is subject to recall bias because it may be affected by bias on mothers self-reported behavior $[5,6]$.

These studies often failed to account for dose-response effects [7]. The dose-to-mother deuterium dilution technique has made it possible to distinguish between water ingested by the baby via breast feeding and water from sources other than human milk. This study allows an objective evaluation of whether a mother is exclusively breast feeding her infant as well as the volume of human milk and water intake. We determined Plasmodium falciparum parasitaemia prevalence in breastfed children aged from 0 to 6 months according to breast feeding regimens and a relation with ingested breastmilk dose evaluated with deuterium dilution technique in southern Benin. 
Citation: de Tove YSS, Ogouyemi-Hounto A, Gilles Cotrell, Alao JM, Hounkpatin AW, et al. (2018) Prevalence of Plasmodium falciparum Parasitaemia in Children from Different Breastfeeding Regimens Evaluated by the Deuterium Technique in Southern Benin. J Trop Dis, 6: 258 6: 258. doi:10.4172/2329-891X.1000258

Page 2 of 6

\section{Materials and Methods}

\section{Study sites}

A cross sectional study was performed between February to December 2014, primarily during the rainy season, in four hospitals, namely the health centers of Ouidah, Pahou, Kindji and Tokpadomè, all located in the sanitary health zone of Ouidah Kpomassè ToriBossito (OKT) a mesoendemic area. Biological analyses were carried out in the Laboratory of Centre de Lutte Intégrée contre le Paludisme (CLIP) located in Cotonou.

\section{Subjects}

\section{Sampling and inclusion criteria}

The sample size has been calculated by the Schwartz formula using the prevalence of Plasmodium falciparum infection (7.8\%) obtained in the OKT health region in children under 12 months [7] and has yielded a total of 110 children. We used this sampling size calculation for children from 0 to 6 months in this study.

During the first 2 weeks of the study, all lactating mothers attending the pediatric unit of follow-up and counselling, who agreed to sign the consent form, were included in the study. All Mother-Child Pairs (MCP) and who met the eligibility criteria were enrolled into the study.

\section{Inclusion criteria included:}

- Resided for at least 6 months in the OKT area;

- HIV negative serologic status;

- Hemoglobin level greater than $7 \mathrm{~g} / \mathrm{dl}$;

- Agreed and signed the consent form.

\section{Training of field workers and data collection}

Before the actual survey, training was organized by nutrition specialists from the International Atomic Energy Agency (IAEA)and parasitology team from Faculté des Sciences de la Santé de Cotonou (Benin) on the purpose, measurement techniques, and the questionnaire (on clinical and epidemiological characteristics, infant health status, malaria prevention with Sulfadoxine-Pyrimethamine (SP) use and long lasting insecticidal nets (LLINs) use. The informed mothers who gave their consent and met the inclusion criteria participated to the study. Each MCP responded to a questionnaire and benefited from a measurement of the anthropometric. Each child received a clinical examination with temperature and a biological check-up (qPCR and quantitative breast milk by oxyde de deuterium).

Variables studied were demographic (age and sex), nutritional (weight, height, body mass index), epidemio-clinics (a fever with a temperature above $37.5^{\circ} \mathrm{C}$ ), taking of at least one dose of SulfadoxinePyrimethamine, and use of the long-lasting insecticidal nets or LLINSs.

The SP use for malaria prevention during pregnancy as intermittent preventive treatment in pregnancy (IPTp), child HIV status, and child's birthweight were recorded in the child's health record and in the maternity register of the health centers. Taking the temperature and looking for an abnormality of the physical examination was carried out respectively by nurses and a pediatrician.

\section{Anthropometry}

Measurements were made by trained personnel using standard procedures [8,9]. Wearing minimal clothes, the mothers were weighed to the nearest $10 \mathrm{~g}$ with an electronic scale (Seca, Hamburg, Germany). Height was measured to the nearest millimeter with gauge of Shorr. The body mass index (BMI) was calculated as weight $(\mathrm{kg})$ over height squared $\left(\mathrm{m}^{2}\right)$. The children were weighed without clothes using a portable electronic infant scale accurate to $5 \mathrm{~g}$. Length was measured using a standardized infantometer of Shorr.

Assuming the recommended cut-offs for data exclusion, data were excluded if a child's length-for-age z-score (LAZ) was below -6 or above +6 , weight-for-age $z$-score (WAZ) was below -6 or above +5 , or weight-for length $\mathrm{z}$-score (WLZ) was below -5 or above +5 , as these extreme values were most likely a result of errors in measurement or data entry [10]. All children admitted for pediatric consultation, and who met the inclusion criteria, were tested for $P$. falciparum in a blood specimen that was collected and sent to the CLIP Laboratory at Cotonou.

\section{Laboratory Examinations}

\section{Capillary blood sampling}

Sample of blood in the heel of the child is extracted: one drops to perform hemoglobin test, and the remaining three drops of blood (50 $\mu \mathrm{L} \times 3$ ) on Whatman $3 \mathrm{MM}$ filter paper. The dried blood impregnated filter papers were stored at $-20^{\circ} \mathrm{C}$ (under conditions of good quality) in a sealed film with silica after complete air drying until DNA extraction and $\mathrm{qPCR}$ in real time was achieved.

\section{Haemoglobin measurement}

Finger-picked blood sample was used to measure hemoglobin using a portable spectrophotometer (Hemo-Control, EKF-Diagnostic, Germany). The positive control micro-dish was used to control the device. From a drop of blood collected at the tip of the finger and deposited on the micro-cuvette (then introduced into the apparatus), the hemoglobin level is measured and displayed in 60 seconds [11]. Individuals found with Plasmodium parasitaemia or hemoglobin level less than $7 \mathrm{~g} / \mathrm{dL}$ were referred to the pediatrician for evaluation and treatment.

\section{The dose-to-the-mother deuterium-oxide technique}

The amount of breast milk consumed by the infant over a period of 14 days was evaluated using the deuterium oxide technique "dose to the mother". This method consists in giving the mother to drink deuterium oxide, then to follow its disappearance through saliva in the mother and its appearance in infants' saliva [12]. In the laboratory, a double reading by the FTIR reading was made for each saliva sample by experienced technicians of the CLIP Laboratory.

The dose-to-mother deuterium dilution technique was used to evaluate the human milk intake, the water from sources other than human milk and the exclusivity of breastfeeding [13-16]. The data about non-breast milk water intake were grouped into three regimens of breastfeeding according to the study reported [17], exclusive breastfeeding $(\mathrm{EBF})=$ non-breast milk water intake $<52 \mathrm{~mL} /$ day; predominant breastfeeding $\quad($ preBF $)=$ non-breast milk water intake $=52-216 \mathrm{~mL} /$ day and partial breastfeeding $($ parBF $)=$ non-breast milk water intake $>216 \mathrm{~mL} /$ day). 
Citation: de Tove YSS, Ogouyemi-Hounto A, Gilles Cotrell, Alao JM, Hounkpatin AW, et al. (2018) Prevalence of Plasmodium falciparum Parasitaemia in Children from Different Breastfeeding Regimens Evaluated by the Deuterium Technique in Southern Benin. J Trop Dis, 6: 258 6: 258. doi:10.4172/2329-891X.1000258

Page 3 of 6

A baseline sample of $2 \mathrm{~mL}$ of saliva from the mother and the child was collected on day 0 , after which, the mother received an oral dose $(30 \mathrm{~g})$ of deuterium oxide $(99.9 \%$ purity; Sercon-Cortec Cambridge Isotope Laboratories Inc, Andover, MA, USA). Further, 12 saliva samples were collected from the mother and the infant on days 1, 2, 3, 4,13 and 14. Saliva samples were stored in ice during transport on the days of collection, brought to the laboratory and stored at $-20^{\circ} \mathrm{C}$ until analysis. Enrichment of saliva samples was measured using a Fourier transform infrared spectrometer (IR-PRESTIGE 21; Shimadzu, Vienna, Austria) with calcium fluoride cell of $0.1 \mathrm{~mm}$ path length. Before measuring deuterium in saliva, the calibration procedure involved preparation of $\mathrm{D} 2 \mathrm{O}$ calibrator by dilution of $\mathrm{D} 2 \mathrm{O}$ with deionized water. The infrared spectra were measured in the range 2300-2900 $\mathrm{cm}^{-1}$.

Magnitude of the response obtained from the FTIR was deducted from the D2O absorption curve by an algorithm (isotope software) developed by the Medical Research Council (MRC; Human Nutrition Research, Cambridge, UK). Intake of breastmilk and water from nonmilk sources was calculated by fitting the deuterium enrichment, anthropometric data, MCP ages to a model for water turnover in the mothers and infants, and the transfer of milk from mother to the infant $[13,14]$ using an algorithm [Excel (2007) spreadsheet developed by the MRC] and clinical information.

\section{Real-time quantitative PCR}

DNA extraction was performed using Chelex 100 technique from the filter papers. Real-time duplex qPCR was performed using primers and probes specific to Plasmodium $\mathrm{spp} / P$. falciparum for the gene encoding the small (18S) subunit of Plasmodium rRNA. This technique was used to test the 115 samples of children breastfed, as described [18]. The samples were subjected to 40 cycles of amplification in the real-time PCR system ViiA 7 (Applied Biosystem) at the Molecular Biology Laboratory of (CLIP) in the Faculté des Sciences de la Santé in Cotonou.

A positive control signal for the human GlycerAldehyde-3Phosphate De-Hydrogenase (GAPDH) gene was amplified for all samples (control of good performance of DNA extraction from the filter paper). To declare that a sample was $P$. falciparum infection positive, the $\mathrm{Ct}$ should be: Plasmoprobe $<40$ cycles, Plasmodium falciparum $<37$ cycles, Ct GAPDH $<40$ cycles, the Ct of $P$. falciparum $3 \mathrm{D} 7<23$ and the negative control must be undetectable. The parasites were quantified using a standard range of DNA made from an in vitro culture suspension of a $P$. falciparum 3D7 line offered by IRD/NIH: $(P$. falciparum 3D7 GL, MRA-1001) filed by Megan Dowler, (Walter Reed Army Institute of Research). Children testing positive were traced and treated according to national guidelines or referred to a local health facility.

\section{Data interpretation and analysis}

P. falciparum infection was defined as positivity of $\mathrm{Qpcr}(\mathrm{Ct}<37)$. For an episode of malaria, there is more a fever. The use of LLINSS is verified by the nurse who had the contact of each CMPs. The use of SP is verified in the register of hospital. The HIV status of each child was sought in the registry. Data were handled and analyzed using Excel software and STATA version 12 (Stata Corp, College Station, TX, USA). Descriptive analyses were done by determining means, and standard deviations. Variables studied were demographic (age and sex), nutritional (weight, height, body mass index), epidemio-clinics (a fever with a temperature above $37.5^{\circ} \mathrm{C}$, taking at least one dose of Sulphadoxine-Pyrimethamine or SP, the use of the long-lasting insecticidal nets or LLINS, positivity of $\mathrm{qPCR}$ ). For quantitative variables, averages and standard deviations were calculated. $\mathrm{p}<0.05$ is considered statistically significant.

\section{Ethics statement}

The Ethical Committee of the Faculté des Sciences de la Santé gave the ethical approval $\mathrm{N}^{\circ} 0103 / 2014 / \mathrm{CE} / \mathrm{FSS} / \mathrm{UAC}$ du03/03/2014 for the study. The study was approved by the health district of OKT authorities. Written informed consent was obtained from the mother of all the children enrolled in the study.

\section{Results}

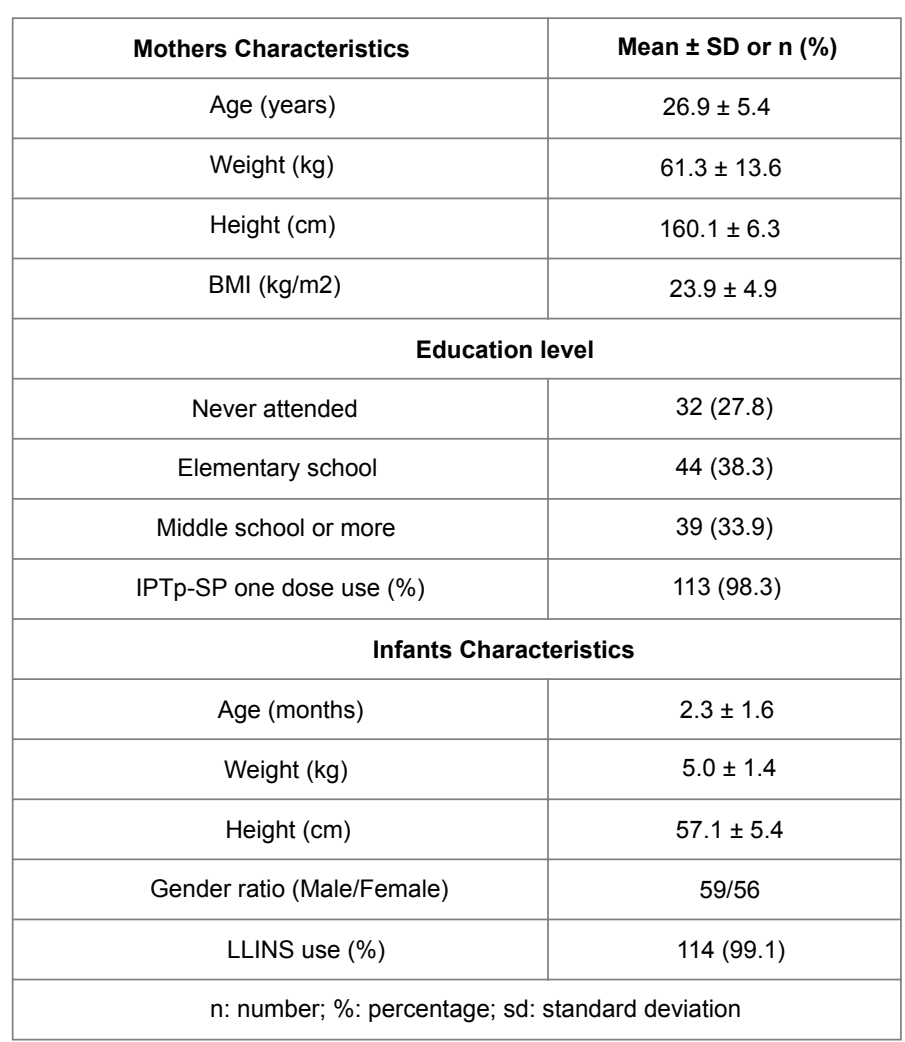

Table 1: Characteristics of mothers and children

A total of 115 pairs of mothers and child were recruited. The average age of the mothers was 26.9 years, with values going from 18 to 40 . Their average weight and height were respectively $61.5 \mathrm{~kg}$ and $161 \mathrm{~cm}$. The average Body Mass Index (BMI) was $23.9 \mathrm{~kg} / \mathrm{m} 2$ with measurements between normal values 18.5 and $25.66 .09 \%$ of the women had at most the elementary school education. $99.1 \%$ used the long lasting insecticide treated nets (LLINs) and 98.3\% benefited from Intermittent Preventive Treatment in pregnancy (IPTp) by using at least one dose of sulfadoxine-pyrimethamine (SP). The children had an average age of 2.3 months. The gender ratio was balanced ( 59 boys and 56 girls), and their weight was $5.0 \mathrm{~kg}$ in average as shown in Table 1. 
Citation: de Tove YSS, Ogouyemi-Hounto A, Gilles Cotrell, Alao JM, Hounkpatin AW, et al. (2018) Prevalence of Plasmodium falciparum Parasitaemia in Children from Different Breastfeeding Regimens Evaluated by the Deuterium Technique in Southern Benin. J Trop Dis, 6: 258 6: 258. doi:10.4172/2329-891X.1000258

Page 4 of 6

\section{Plasmodium falciparum parasitaemia prevalences}

The distribution of Plasmodium falciparum parasitaemia according the breast feeding practices is presented in Table 2.

\begin{tabular}{|c|c|c|c|}
\hline Breast feeding regimen & $\mathbf{N}$ & $\mathbf{n}$ & Prevalence (\%) \\
\hline Exclusive & 4 & 0 & 0 \\
\hline Predominant & 59 & 3 & 5.08 \\
\hline Partial & 52 & 2 & 3.85 \\
\hline Total & 115 & 5 & 4.35 \\
\hline
\end{tabular}

$\mathrm{N}$ : number examined; $\mathrm{n}$ : number of parasitaemia positive; \%: prevalence of parasitaemia

Table 2: Plasmodium falciparum parasitaemia in different breastfeeding regimens at OKT health area

Of the 115 children examined, 5 were infected with Plasmodium falciparum identified by qPCR, giving a global prevalence of $4.35 \%$ with $0 \%$ in EBF regimen, 5.08\% (3/59) in PreEBF regimen, and 3.85\% $(2 / 52)$ in ParEBF regimen.

\section{Average quantity of breast milk and water absorbed by the child}

Table 3 shows the average quantity of breast milk and water absorbed by the child. The average amount of breastmilk and water ingested by children from 0 to 3 month were respectively 587.68 and $729.05 \mathrm{ml}$ per day.

\begin{tabular}{|c|c|c|c|}
\hline Age (month) & N & Maternal milk (mL day) & Non maternal water (mL day) \\
\hline $0-3$ & 71 & 587.68 & 223 \\
\hline 03-Jun & 44 & 729.05 & 308.7 \\
\hline \multicolumn{3}{|c|}{ N: number of children; Cl: Confidence Interval } \\
\hline
\end{tabular}

Table 3: Quantities of maternal milk and non-breast water ingested by children according to age range

\section{Relation between the quantity of milk taken and Plasmodium falciparum}

Table 4 presents the relation between the quantity of milk taken and Plasmodium falciparum parasitaemia adjusted to age. There is a statistically significant difference between the average quantity of breastmilk taken by the infected (Plasmodium falciparum parasitaemia with qPCR positive) children and children without parasitaemia. The infected children have taken about $164.11 \mathrm{~mL}$ of breastmilk less than the children who were not infected. Children of age of 3 months or less have taken an average $140.9 \mathrm{~mL}$ of breastmilk less than the children who are older than 3 months.

\begin{tabular}{|c|c|c|c|c|}
\hline $\begin{array}{c}\text { Quantity of maternal } \\
\text { milk (n) }\end{array}$ & Coefficient & Cl \% & p value \\
\hline \multicolumn{5}{|c|}{ PCR } \\
\hline negative & 110 & 0 & - & 0 \\
\hline positive & 5 & -164.11 & $-234.45 ;$ & \\
\hline
\end{tabular}

\begin{tabular}{|c|c|c|c|c|}
\hline & & & -93.76 & \\
\hline \multicolumn{5}{|c|}{ Age } \\
\hline$\geq 3$ months & 44 & 0 & - & \multirow[b]{2}{*}{0} \\
\hline$<3$ months & 71 & -140.88 & $\begin{array}{c}-214.87 \\
-66.89\end{array}$ & \\
\hline \multicolumn{5}{|c|}{$\mathrm{Cl}$ : Confidence interval } \\
\hline
\end{tabular}

Table 4: Relation between quantity of breastmilk intake and Plasmodium falciparum parasitaemia (qPCR positive) adjusted on age: a linear regression

\section{Relation between Plasmodium falciparum parasitaemia and breastfeeding regimens}

Table 5 describe the relation between Plasmodium falciparum parasitaemia prevalence and the type of breastfeeding regimen, adjusted on age, mother's parity and IPTp used and LLINs. There is statistical significant with a low risk of Plasmodium falciparum when the mother had IPTp and used LLINs. Maternal parity and was not significantly associated to malaria infection in this study.

\begin{tabular}{|c|c|c|}
\hline \multicolumn{3}{|c|}{ Plasmodium falciparum parasitaemia } \\
\hline Eclusive and predominant breastfeeding & Odds Ratio ( $\mathrm{Cl} \%)$ & $\begin{array}{c}p \\
\text { value }\end{array}$ \\
\hline No & 1 & \multirow{2}{*}{0.772} \\
\hline Yes & $1.44(0.11-17.58)$ & \\
\hline \multicolumn{3}{|l|}{ Age } \\
\hline$\geq 3$ months & 1 & \multirow{2}{*}{0.496} \\
\hline$<3$ months & $2.47(0.18-33.62)$ & \\
\hline \multicolumn{3}{|l|}{ Parity } \\
\hline$<2$ & 1 & \multirow{2}{*}{0.573} \\
\hline$\geq 2$ & $0.49(0.042-5.73)$ & \\
\hline \multicolumn{3}{|c|}{ IPTp one dose } \\
\hline No & 1 & \multirow{2}{*}{0.05} \\
\hline Yes & $0.034(0.00-1.05)$ & \\
\hline \multicolumn{3}{|l|}{ LLINs } \\
\hline No & 1 & \multirow{2}{*}{0.04} \\
\hline Yes & $0.017(0.00-0.60)$ & \\
\hline \multicolumn{3}{|c|}{$\mathrm{Cl}=$ Confident interval } \\
\hline
\end{tabular}

Table 5: Relation between Plasmodium falciparum parasitaemia and breastfeeding regimens adjusted to others variables (age, parity, IPTp, LLINs): a logistic regression.

\section{Discussion}

The aim of our study was to determine Plasmodium falciparum parasitaemia prevalence in children aged less than six month according to their breastfeeding regimen and the link between maternal milk 
Citation: de Tove YSS, Ogouyemi-Hounto A, Gilles Cotrell, Alao JM, Hounkpatin AW, et al. (2018) Prevalence of Plasmodium falciparum Parasitaemia in Children from Different Breastfeeding Regimens Evaluated by the Deuterium Technique in Southern Benin. J Trop Dis, 6: 258 6: 258. doi:10.4172/2329-891X.1000258

Page 5 of 6

dose ingested and Plasmodium falciparum parasitaemia in southern Benin. With the quantitative techniques used, we found a low Plasmodium falciparum parasitaemia prevalence and a significant relation between Plasmodium falciparum parasitaemia and the amount of milk ingested in our study. No study carried out in vivo had previously suggested a relationship between both.

In our study, the average age of infants was 2.3 months, similar to the Medoua research in 2007 which studied infants aged between 1.15 and 4.5 months with an average age of 3 months [12]. In Haisma study's, infants were 4 months old and an average age of 2.1 months [17]. In a study conducted in Cameroon, however, the population was $65.9 \%$ female. Beninese mothers had a normal average body mass index (BMI) $\left(23.9 \mathrm{~kg} / \mathrm{m}^{2}\right)$. During pregnancy, they had taken at least one dose of SP, which would reduce serious placental parasitism. The use of LLINSs was high during the study (92\%) different from the frequency observed in the study conducted by combien in the OKT zone [8].

The overall prevalence of malaria among the 115 infants aged less than 6 months who were breastfed in our study was $4.35 \%$. It is lower than that found by Brazeau in the Congo children (10.5\%). It is necessary to stipulate that the techniques used have precisions. The deuterium oxide "dose to the mother" allowed us to determine the amount of breastmilk and the amount of fluid consumed from sources other than breastmilk, which makes it possible to define in a very precise way the breastfeeding regimens. These quantitative results are not affected by recalls, found during mothers' declarations $[5,6]$. This technique classifies breastfeeding regimens into exclusive, predominant and partial types.

However, the classification of the type of breastfeeding using the deuterium oxide technique depends on the volume of non-dairy water consumed by the infant. This parameter should be standardized and associated with the WHO classification of breastfeeding regimen [17]. We also identified the Plasmodium falciparum parasitaemia prevalence by qPCR. It appeared in our study that only $3.48 \%$ of mothers breastfed their babies exclusively. The majority was classified as mainly predominant $(51.30 \%)$ or partial $(45.22 \%)$.

Regarding the relationship between breastfeeding and Plasmodium falciparum parasitaemia there was no significant association. In fact, exclusive breastfeeding is not an essential method of providing protection [18]. In fact, it is possibly due to a dose-response effect [7]. By examining the relationship between the amount of milk ingested and malaria infection, the risk of malaria infection increased when the amount of breast milk decreased significantly $(p=0.00$, CI-234.45-93.76). The protection against malaria, therefore, depends on the amount of milk ingested by the infant. According to Lartey study, infants who consume a significant amount of non-dairy water and a large amount of food other than breast milk are more susceptible to infections [19].

The relationship between malaria infection and breastfeeding regimen adjusted for age, parity, IPT and LLINS covariates shows that IPTp $(\mathrm{p}=0.05)$ and LLINS $(\mathrm{p}=0.04)$ were significant $\mathrm{a})$ with a reduced risk for the child to have Plasmodium falciparum parasitaemia when the mother has received at least one dose of SP during IPT or has slept under LLINS.

Appropriate statistical methods were used to account for the crosssectional nature of the data. Our study reveals a potential protective effect of breast milk on Plasmodium falciparum parasitaemia. Predominant breastfeeding supplemented with small amounts of water porridge or herbal tea does not eliminate the protection offered by breast milk. Obviously, this is not meant to discourage exclusive breastfeeding, but in our society where the mother had to take on many responsibilities in addition to feeding their babies, exclusive breastfeeding is not always practiced. Finally, breastfeeding does not obey to the law of "all or nothing". The more breastmilk an infant receives in the first six months of life, the better protected he will be.

The limits of our study are related to the size of our sample. Due to financial limitations, the size of the sample could not be increased. Thus, breastfeeding practices reflect the inherent differences between mothers. Finally, the sample size of this study did not allow us to examine the effect of exclusive breastfeeding regimen alone because the size of the population of this group does not allow such a study. We therefore, grouped the exclusive and predominant breastfeeding group during the analysis. Exclusive breastfeeding may provide breastmilk protection against malaria because of the duration of breastfeeding and the amount of milk ingested by the infant in exclusive regimen.

\section{Conclusion}

The prevalence global prevalence of Plasmodium falciparum parasitaemia was $4.35 \%$. Our findings suggest that exclusive breastfeeding may reduce the risk of $P$. falciparum in southern Benin, in addition to the use of LLINS in young infants and IPTp in mothers. Study with larger numbers of patients, conducted for a longer time would be necessary to confirm this relationship between malaria and quantity of ingested breast milk.

\section{Source of Funding}

The study was part of the International Atomic Energy Agency, Technical Cooperation Project $\mathrm{N}^{\circ} \mathrm{CT} / \mathrm{Ben}$ 6/005 and Benin Ministry of Health.

\section{Conflicts of Interest}

The authors declare that they have no conflicts of interest.

\section{Contributions}

All authors participated in the design and planning of the study. YSSDT, AOH, JMA, AWH, GD, AM, DK, PB, AA, CF, DA, KK, CA, NGM participated to the collect of data in the field and laboratory work. YSSDT, GC and LA analysed data. All authors reviewed the manuscript and approved the final version.

\section{Acknowledgements}

The authors wish to thank OKT women who generously gave their time and commitment to fulfill the requirements for participating in this study, Dr. Savi de Tove Stephane KM, Mrs Slater Christine, Cornelia Loechl, Adeline Djeutie from IAEA.

\section{References}

1. Nankabirwa V, Tylleskar T, Nankunda J, Engebretsen IMS, Sommerfelt $\mathrm{H}$, et al. (2011) Promise EBF Research Consortium. Malaria parasitaemia among infants and its association with breastfeeding peer counselling and vitamin A supplementation: a secondary analysis of a cluster randomized trial. PLoS One 6: e21862. 
Citation: de Tove YSS, Ogouyemi-Hounto A, Gilles Cotrell, Alao JM, Hounkpatin AW, et al. (2018) Prevalence of Plasmodium falciparum Parasitaemia in Children from Different Breastfeeding Regimens Evaluated by the Deuterium Technique in Southern Benin. J Trop Dis,

Page 6 of 6

2. Ibadin O, Ofili N, Morrison O, Nkwuo E (2012) Exclusive breastfeeding and malaria in early infancy: Experience from Benin City, Nigeria. J Med Biomed Res 11: 116-122.

3. Kalanda BF, Verhoeff FH, Brabin BJ (2006) Breast and complementary feeding practices in relation to morbidity and growth in Malawian infants. Eur J Clin Nutr 60: 401-440.

4. Brazeau NF, Tabala M, Kiketa L, Kayembe D, Chalachala JL, et al. (2016) Exclusive breastfeeding and clinical malaria risk in 6-month-old infants: A cross-sectional study from kinshasa, democratic republic of the Congo. Am J Trop Med Hyg 95: 827-830.

5. Hebert JR, Yunsheng M, Clemow L, Ockene IS, Saperia G, et al. (1997) Gender differences in social desirability and social approval bias in dietary self-report. Am J Epidemiol 146: 10461055.

6. Piwoz EG, Kanashiro KH, Lopez RG, Black RE, Brown H, et al. (1995) Potenial for misclassification of infant's usual feeding pratices using 24 hour dietary assessment methods. J Nutr 125: 57-65.

7. Scariati PD, Grummer SLM, Beck FS (1997) Longitudinal analysis of infant morbidity and the extent of breastfeeding in the United States. Pediatrics 99: 99-105.

8. Damien GB, Djènontin A, Rogier C, Corbel V, Bio BS, et al. (2010) Malaria infection and disease in an area with pyrethroid- resistant vectors in southern Benin. Malar J 9: 380.

9. Cogill B (2003) Anthropometric indicators guide. Technical Assistance Project for Food and Nutrition. Academy for Educational Development, Washington, DC.

10. World Health Organization (2006) Child growth standards: Length/ height-for-age, weight-for-age, weight-for-length, weight-for-height and body mass index-for-age: Methods and development. WHO, Geneva.

11. Aakre K, Bliksrud Y, Frøyshov B, Hager H, Hansen A, et al. (2009) Validation of the hemo control instrument for determination of $\mathrm{B}$ - haemoglobin concentration in primary health care. Scand J Clin Lab Invest 69: 817-821

12. Medoua GN, Nana ECS, Ndzana ACA, Makamto CS, Etame LS, et al. (2011) Breastfeeding practices of Cameroonian mothers determined by dietary recall since birth and the dose-to-the-mother deuterium-oxide turnover technique. Maternal and Child Nutr 35: 698-702.

13. Coward WA, Cole TJ, Sawyer MB, Prentice AM (1982) Breast-milk intake measurement in mixed-fed infants by administration of deuterium oxide to their mothers. Hum Nutr Clin Nutr 36: 141-148.

14. Ewing AK, Heywood PF, Coward WA (1986) Longitudinal measurements of breast milk output by a $2 \mathrm{H} 2 \mathrm{O}$ tracer technique in rural Papua New Guinean women. Human Nutr Clin Nutr 40: 451-467.

15. Butte NF, Wong WW, Patterson BW, Garza C, Klein PD, et al. (1988) Human-milk intake measured by administration of deuterium oxide to the mother: a comparison with the test-weighing technique. Am J Clin Nut 47: 815-821.

16. Cisse SA, Bluck L, Diaham B, Dossou N, Guiro TA, et al. (2002) Use of Fourier transformed infrared spectrophotometer (FTIR) for determination of breastmilk output by the deuterium dilution method among Senegalese women. F Nutr Bull 23: 138-141.

17. Haisma H, Coward WA, Albernaz E, Visser GH, Wells JCK, et al. (2003) Breast milk and energy intake in exclusively, predominantly and partially breast-fed infants. Eur J Clin Nutr 57: 1633-1642.

18. Diallo A, Ndam NT, Moussiliou A, Dos SS, Ndonky A, et al. (2012) Asymptomatic carriage of Plasmodium in urban Dakar: The risk of malaria should not be underestimated. PLoS One 7: e31100.

19. Lartey A, Manu A, Brown KH, Peerson JM, Dewey KG, et al. (2000) Predictors of growth from 1 to 18 months among breast-fed Ghanaian infants. Euro J Clin Nutr 54: 41-49. 